\title{
Tunable seesaw-like 3D capacitive sensor for force and acceleration sensing
}

\author{
Jilong Ye $\mathbb{D}^{1,2,8}$, Fan Zhang $\mathbb{D}^{3,4,8 凶}$, Zhangming Shen $\mathbb{D}^{3,4}$, Shunze Cao ${ }^{3,4}$, Tianqi Jin $\mathbb{D}^{3,4}$, Xiaogang Guo ${ }^{5}$, Zhihong Li ${ }^{2,6}$, Li Lin $\mathbb{D i D}^{7 凶}$ and \\ Yihui Zhang id $3,4 \bowtie$
}

To address the resource-competing issue between high sensitivity and wide working range for a stand-alone sensor, development of capacitive sensors with an adjustable gap between two electrodes has been of growing interest. While several approaches have been developed to fabricate tunable capacitive sensors, it remains challenging to achieve, simultaneously, a broad range of tunable sensitivity and working range in a single device. In this work, a 3D capacitive sensor with a seesaw-like shape is designed and fabricated by the controlled compressive buckling assembly, which leverages the mechanically tunable configuration to achieve high-precision force sensing (resolution $\sim 5.22 \mathrm{nN}$ ) and unprecedented adjustment range (by $\sim 33$ times) of sensitivity. The mechanical tests under different loading conditions demonstrate the stability and reliability of capacitive sensors. Incorporation of an asymmetric seesaw-like structure design in the capacitive sensor allows the acceleration measurement with a tunable sensitivity. These results suggest simple and low-cost routes to high-performance, tunable 3D capacitive sensors, with diverse potential applications in wearable electronics and biomedical devices.

npj Flexible Electronics (2021)5:28; https://doi.org/10.1038/s41528-021-00125-9

\section{INTRODUCTION}

With the rapid development of information technology and global informationization, a large number of miniaturized sensors have been used in different aspects of daily life and engineering fields ${ }^{1-4}$. Among various types of sensors, the force sensors with high sensitivity and high dynamic range, are widely demanded in the applications of health monitoring ${ }^{5-8}$, electronic devices ${ }^{9-13}$, and robotics ${ }^{14-16}$. Recent advances in fabrication and assembly techniques enable the applications of force sensors in noninvasive heart rate and pulse monitoring ${ }^{17-19}$, skin health monitors ${ }^{20,21}$, implantable devices ${ }^{22-24}$, accelerometer ${ }^{25,26}$, electronic skin ${ }^{27-30}$, and haptic feedback ${ }^{31-33}$. For example, Han et al. developed a wireless sensing system to detect the pressure of the skin-prosthetic interface based on the resistive sensor ${ }^{20}$. This resistive sensor was also integrated into a minimally invasive surgical instrument to improve surgical performance and patient outcomes $^{22}$. Additionally, the sensors formed by various materials and constructions have been designed and fabricated to achieve force sensing ${ }^{34-40}$.

Compared with piezoresistive sensors ${ }^{34,41,42}$, piezoelectric sensors $^{28,35,43}$, and optical sensors ${ }^{36,44}$, the capacitive sensors have the advantages of high sensitivity, good dynamic response, and low

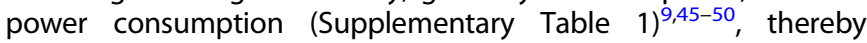
facilitating measurements of pressure, displacement, acceleration, etc. Typically, a capacitive force sensor contains a fixed plate and a movable plate forming the force sensing diaphragm. The sensitivity of parallel-plate capacitive sensors is determined by $\varepsilon_{0} \varepsilon_{\mathrm{r}} A / d^{2}$, where $A$ and $d$ are the overlapping area and the gap between two electrodes, respectively; $\varepsilon_{\mathrm{r}}$ is the relative permittivity of material; and $\varepsilon_{0}$ is vacuum permittivity ${ }^{51}$. Based on this relationship, the customized designs of capacitive sensors can be achieved easily. For instance, the sensitivity of capacitive sensors can be improved by designing the overlapping area or the gap rationally. Despite the advantages of capacitive sensors, the sensitivity of most stand-alone capacitive sensors is fixed, including those based on the fabrication process of micro-electromechanical systems (MEMS), which limits the application range of capacitive sensors $^{52,53}$. For example, the elastic modulus of liver macrophages of mice usually undergoes a significant change after the chemical attack ${ }^{54}$. In order to achieve the real-time recording of the varying elastic modulus, a stand-alone sensor with both high sensitivity and wide working range is required. The capacitive sensor with a wide tunability is also promising to address the resourcecompeting issue between high sensitivity and wide working range. A few different strategies have been developed to achieve tunable capacitive sensors, such as those based on microstructure design and dielectric materials ${ }^{55-58}$. Combined with MEMS technologies, the capacitive sensing systems with tunable sensitivity were demonstrated, which involved complicated designs (e.g., four degrees of series-parallel resonator array) and fabrication processes (e.g., high temperature, high pressure, and face the potential risk of structure adhesion due to capillarity) ${ }^{56,57}$. Incorporating dielectric elastomers that can deform under an applied electric field as the dielectric layer of the tunable capacitive sensor allows the variation of electrode gap, but requires high voltages and complicated electric circuits ${ }^{55}$. Up to now, the development of capacitive sensors that combine a high tunability of sensitivity and a wide working range is still highly desired.

In this paper, we introduce a design and fabrication scheme of 3D capacitive sensors, based on the mechanics-guided deterministic assembly ${ }^{37,59-68}$, to overcome the above limitations. The proposed strategy enables the formation of 3D capacitive sensors

\footnotetext{
${ }^{1}$ State Key Laboratory of Tribology, Tsinghua University, Beijing 100084, P. R. China. ${ }^{2}$ Center for Nano and Micro Mechanics, Tsinghua University, Beijing 100084, P. R. China. ${ }^{3}$ AML, Department of Engineering Mechanics, Tsinghua University, Beijing 100084, P. R. China. ${ }^{4}$ Center for Flexible Electronics Technology, Tsinghua University, Beijing 100084, P. R. China. ${ }^{5}$ Institute of Advanced Structure Technology, Beijing Institute of Technology, Beijing 100081, P. R. China. ${ }^{6}$ National Key Laboratory of Nano/Micro Fabrication Technology, Institute of Microelectronics, Peking University, Beijing 100871, P. R. China. ${ }^{7}$ Tianjin Research Institute for Advanced Equipment, Tsinghua University, Tianjin 300300, P. R. China. ${ }^{8}$ These authors contributed equally: Jilong Ye, Fan Zhang. ${ }^{凶}$ email: fanzhang252@tsinghua.edu.cn; linli@tsinghua-tj.org; yihuizhang@tsinghua.edu.cn
} 

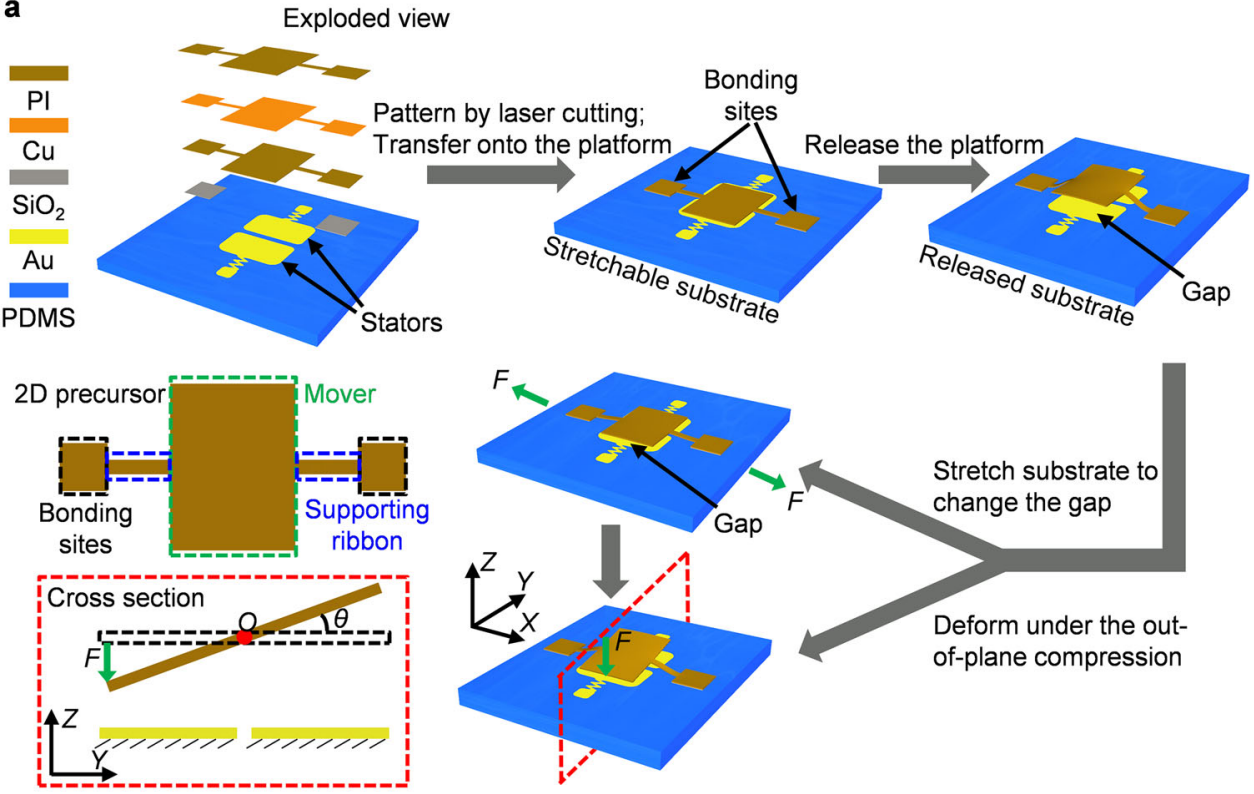

b
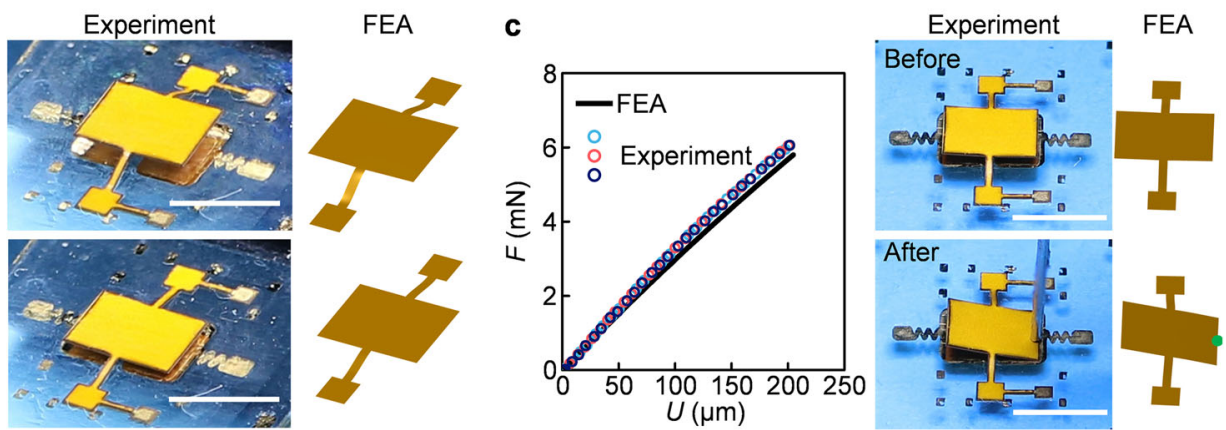

Fig. 1 Design, fabrication, measurements, and simulations of the 3D capacitive sensor through mechanics-guided assembly. a Schematic illustration of fabrication procedures for the 3D capacitive sensor. b Optical images and corresponding FEA predictions of 3D seesaw-like mesostructures in polyimide $(25 \mu \mathrm{m}) / \mathrm{Cu}(25 \mu \mathrm{m}) /$ polyimide $(25 \mu \mathrm{m})$ under different applied strains $\left(\varepsilon_{\text {appl }}=0 \%\right.$ and $\left.3 \%\right)$. c Measured and simulated force responses of the 3D seesaw-like mesostructure. The right panel shows the optical images and FEA predictions of 3D mesostructures before and after out-of-plane compression. Scale bars, $4 \mathrm{~mm}$.

that can adjust the sensitivity and working range rapidly and reversibly among multiple working states, by simply applying external forces on the elastomeric substrate. The computational and experimental studies indicate that the ability of developed 3D seesaw-like mesostructures can change the gap between the electrodes from dozens of micrometers to hundreds of micrometers. Cyclic mechanical tests demonstrate the stability and reliability of this 3D capacitive sensor. Quantitative measurements illustrate the excellent performance of the 3D capacitive sensor, including the force resolution $(\sim 5.22 \mathrm{nN})$ and unprecedentedly tunable range (by $\sim 33$ times) of sensitivity. Furthermore, we introduce an asymmetric design of the 3D capacitive sensor to enable the acceleration measurement with a tunable sensitivity.

\section{RESULTS}

3D capacitive sensor formed by mechanics-guided assembly

Figure 1a illustrates the fabrication procedure of a 3D capacitive force sensor through the mechanics-guided assembly. This 3D differential capacitor consists of two lower electrodes (i.e., two stators in yellow parts) on the elastomeric substrate (polydimethylsiloxane, PDMS) and a 3D seesaw-like mesostructure in polyimide $(\mathrm{PI})$ /copper $(\mathrm{Cu}) /$ polyimide $(\mathrm{PI})$. The two square anchors (in black frames, lower left in Fig. 1a) of the 2D precursor serve as the bonding sites with a prestretched elastomeric substrate, while the other regions (the mover in a green frame and supporting ribbons in blue frames, lower left in Fig. 1a) of the 2D precursor form a weak interface governed by van der Waals interactions. The release of uniaxial prestrain $(\sim 3 \%)$ results in a controlled mechanical buckling that results in the formation of a 3D seesaw-like mesostructure (upper right in Fig. 1a). Consequently, the two stators (lower electrodes) and one mover (upper electrode) form a differential capacitor. The capacitance of this differential capacitor changes due to the variation of the gap between the stators and the mover, when the out-of-plane compressive force $(F)$ is applied on the edge of the mover. After the assembly process, the initial gap $\left(d_{0}\right)$ can still be tuned by applying tensile strain to the elastomeric substrate through the stretching platform with screw micrometers, whose displacement precision is $0.01 \mathrm{~mm}$, corresponding to an applied strain of $\sim 0.144 \%$ (Supplementary Fig. 1). Here, the air is treated as a dielectric with a relative dielectric constant $\left(\varepsilon_{r}=1.0\right)$. Two working states of the 3D capacitive sensor under different levels of applied strain $\left(\varepsilon_{\mathrm{appl}}=0 \%, 3 \%\right)$ are demonstrated in Fig. $1 \mathrm{~b}$, in which the finite element analysis (FEA) predictions and experimental results show good agreements. The scanning electron microscope (SEM) images show the developed 3D capacitive sensor viewed from different angles (Supplementary Fig. 2). Figure 1c presents the 
a

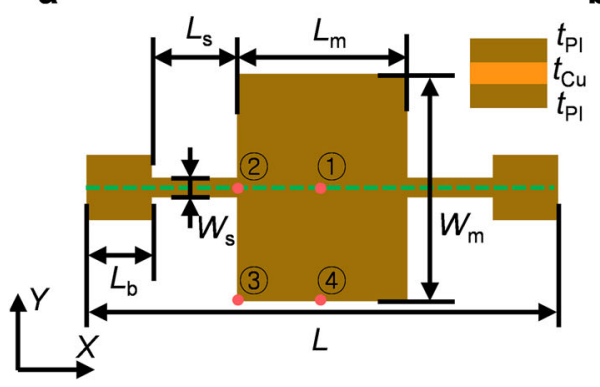

d

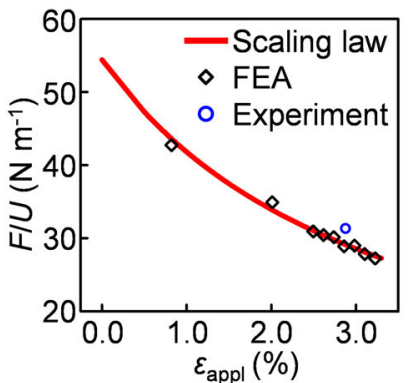

b

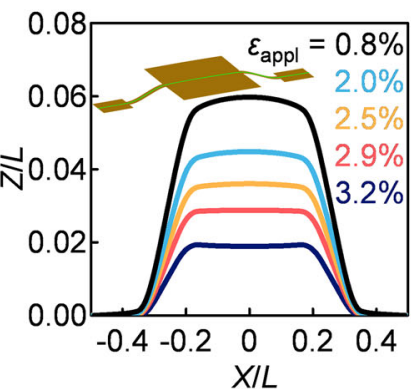

e

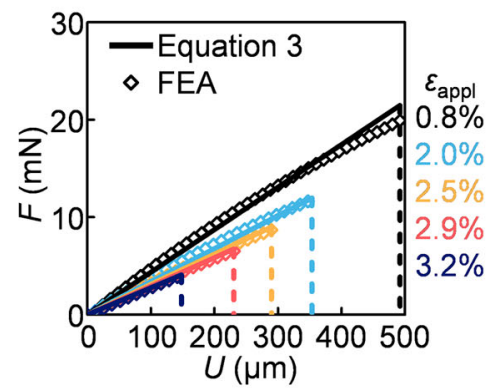

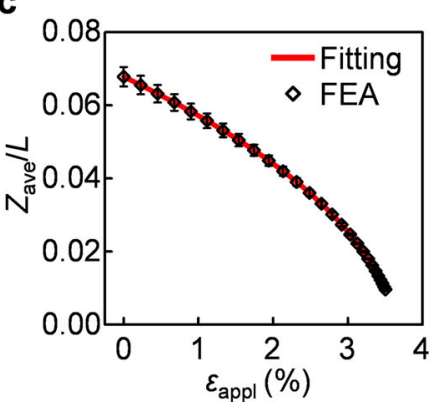

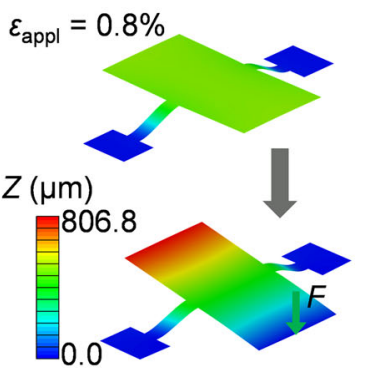

f

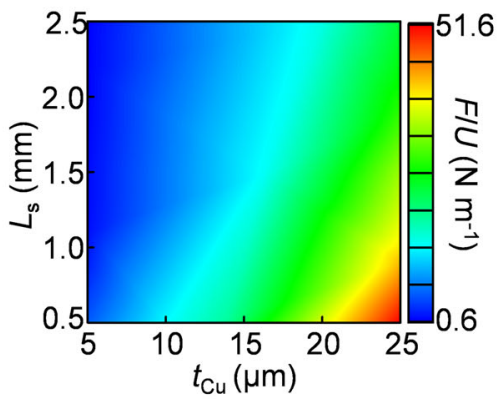

g

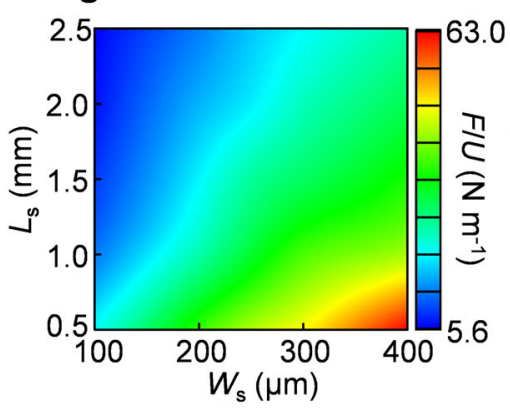

h

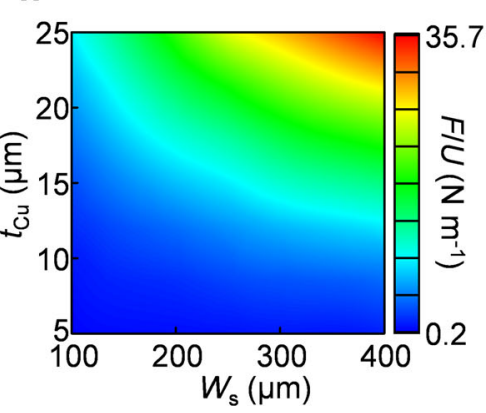

Fig. 2 Mechanical tunability of the 3D seesaw-like mesostructure. a Schematic illustration of geometric parameters of the precursor pattern used to assemble the 3D seesaw-like mesostructure, including thickness $t$; supporting ribbon width $W_{\mathrm{s}}$, and length $L_{\mathrm{s}}$; mover width $W_{\mathrm{m}}$ and length $L_{m} ;$ and the length $L_{b}$ of bonding sites. b Profile of the 3D seesaw-like mesostructure (along with the green line) under different applied strains $\left(\varepsilon_{\mathrm{appl}}=0.8 \%, 2.0 \%, 2.5 \%, 2.9 \%\right.$, and $\left.3.2 \%\right)$. The inset shows the profile of the $3 \mathrm{D}$ mesostructure. c Normalized average height $\left(Z_{\text {ave }} / L\right)$ of four points (point 1 4, shown in (a)) as a function of applied strains $\left(\varepsilon_{\text {appl }}\right)$. d Scaling laws, FEA results, and measurements for the equivalent stiffness (F/U) as a function of applied strain $\left(\varepsilon_{\text {appl }}\right)$. e Out-of-plane compressive force as a function of Z-direction displacement $(U)$ under different applied strain $\left(\varepsilon_{\text {appl }}=0.8 \%, 2.0 \%, 2.5 \%, 2.9 \%\right.$, and $\left.3.2 \%\right)$. The right panel shows the $Z$ coordinate under the applied strain $\left(\varepsilon_{\text {appl }}=0.8 \%\right)$. $f$ Contour plot of the equivalent stiffness $(F / U)$ of the $3 D$ seesaw-like mesostructure in terms of $L_{s}$ and $t_{C u}$ for $W_{s}=350 \mu \mathrm{m}$ and $\varepsilon_{\text {appl }}=1.2 \%$. g Contour plot of the equivalent stiffness $(F / U)$ of the 3D seesaw-like mesostructure in terms of $L_{\mathrm{s}}$ and $W_{\mathrm{s}}$ for $t_{\mathrm{Cu}}=25 \mu \mathrm{m}$ and $\varepsilon_{\mathrm{appl}}=1.2 \%$. $\mathbf{h}$ Contour plot of the equivalent stiffness $(F / U)$ of the $3 D$ seesaw-like mesostructure in terms of $t_{C u}$ and $W_{\mathrm{s}}$ for $L_{\mathrm{s}}=1.5 \mathrm{~mm}$ and $\varepsilon_{\mathrm{appl}}=1.2 \%$.

Table 1. Design parameters of capacitive seesaw-like mesostructures fabricated by the mechanics-guided assembly.

\begin{tabular}{lllllll}
\hline$L(\mathrm{~mm})$ & $t(\mu \mathrm{m})$ & $W_{\mathrm{s}}(\mu \mathrm{m})$ & $L_{\mathrm{s}}(\mathrm{mm})$ & $L_{\mathrm{m}}(\mathrm{mm})$ & $W_{\mathrm{m}}(\mathrm{mm})$ & $L_{\mathrm{b}}(\mathrm{mm})$ \\
\hline 8.3 & 75 & 350 & 1.5 & 3 & 4 & 1.15 \\
\hline
\end{tabular}

compressive force $(F)$ as a function of maximum deflection $(U)$ (i.e., out-of-plane compressive displacement $(U)$ along with the negative Z-direction). In accordance with the FEA results, the measured force varies almost linearly with the displacement, which is similar to that reported from MEMS force sensors ${ }^{69}$. Optical images and corresponding FEA predictions of a 3D seesaw-like mesostructure at the initial state and compressive state $(U \approx 200 \mu \mathrm{m})$ are shown in the right panel of Fig. 1c. The cyclic loading measurements and test protocol are provided in Supplementary Fig. 3.

\section{Mechanical properties of the 3D seesaw-like mesostructure}

The variation of capacitance mainly follows from the torsional deformation of the 3D seesaw-like mesostructure. In this section, we focus on the relationship between mechanical responses of $3 D$ seesaw-like mesostructures and geometrical designs. The 2D precursor of 3D seesaw-like mesostructure is shown in Fig. 2a, which can be characterized by the following geometric parameters, including: uniform thickness $t=2 t_{\mathrm{PI}}+t_{\mathrm{Cu}}$ i supporting ribbon width $W_{\mathrm{s}}$ and length $L_{s} ;$ mover width $W_{\mathrm{m}}$ and length $L_{\mathrm{m}}$; as well as the length of bonding sites $L_{\mathrm{b}}$. The basic design parameters of capacitive seesaw-like mesostructures are listed in Table 1. Figure $2 \mathrm{~b}$ shows the profiles of $3 \mathrm{D}$ seesaw-like mesostructures along with the green line (shown in Fig. 2a) at different levels of applied strain $\varepsilon_{\text {appl }}=0.8 \%, 2.0 \%, 2.5 \%, 2.9 \%$, and $3.2 \%$. The bending deformations mainly occur at supporting ribbons after the buckling assembly, due to the bending stiffness of supporting ribbons (that is much less than that of the mover $\left.\left(W_{\mathrm{s}}<W_{\mathrm{m}}\right)\right)$ and the elastoplasticity of the metal layer. Here, the 
average $Z$ coordinates $\left(Z_{\text {ave }}\right)$ of four points on the mover (point $1 \sim 4$, shown in Fig. 2a) is used to measure the location of the mover. Figure $2 c$ presents the FEA results of the normalized average $Z$ coordinates $\left(Z_{\text {ave }} / L\right)$ of these four points as a function of applied strain, which is fitted by a polynomial $\left(Z_{\text {ave }} / L=\right.$ $\sum_{n=0}^{9} a_{n} \varepsilon_{\mathrm{appl}}^{n}$, the coefficients $a_{n}$ are shown in Supplementary Table 2). With the increase of the applied strain, the normalized average $Z$ coordinate and its standard deviation decrease gradually, which indicates that the mover can be regarded as a plane electrode at different working states. The $Z$ coordinates of mover measured through the white light interferometry are shown in Supplementary Fig. 4. Therefore, the initial gap $\left(d_{0}\right)$ between the mover and stators can be approximately regarded as a constant at the uncompressed state for different levels of applied strain.

During the deformation process under out-of-plane compression, it is reasonable to assume that the displacement of the indenter is equal to the displacement of point 4 . In practical applications, a probe can be adhered at the midpoint of the edge of mover (i.e., point 4) to form a fixed contact point. For relatively small torsion angles $(\theta$, shown in Fig. 1a), the out-of-plane compressive displacement $(U)$ at point 4 is obtained from the geometric analyses as

$U=\frac{W_{\mathrm{m}}}{2} \theta$,

The dimensional analysis suggests that the torsion angle $(\theta)$ of mover is inversely proportional to the torsional stiffness $\left(G W_{s} t^{3}\right)$, and is linearly proportional to the torque $\left(F W_{m} / 2\right)$ as well as the length of supporting ribbon $\left(L_{s}\right)$, which gives

$\theta=a \frac{F W_{\mathrm{m}} L_{\mathrm{s}}}{2 G W_{\mathrm{s}} t^{3}}$

where $G$ is the shear modulus. According to Eqs. (1) and (2), the out-of-plane compressive displacement $(U)$ is linearly proportional to the compressive force $(F)$, i.e.,

$$
U=a \frac{W_{m}^{2} L_{s}}{4 G W_{s} t^{3}} F
$$
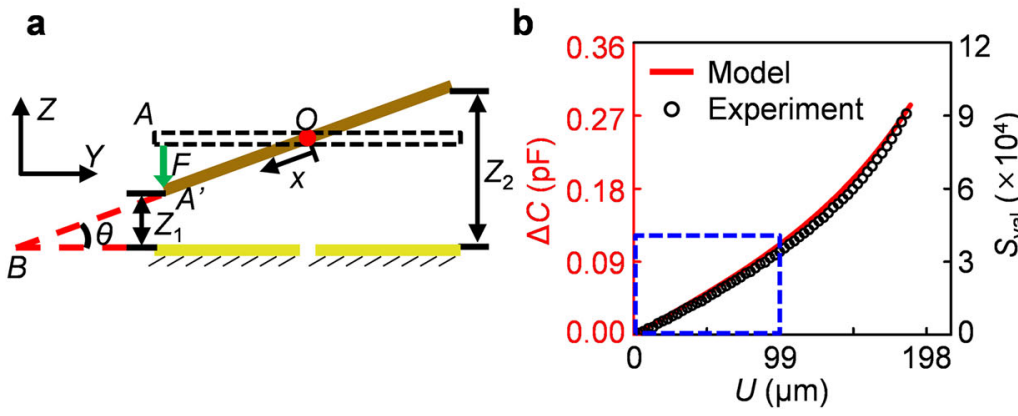

d
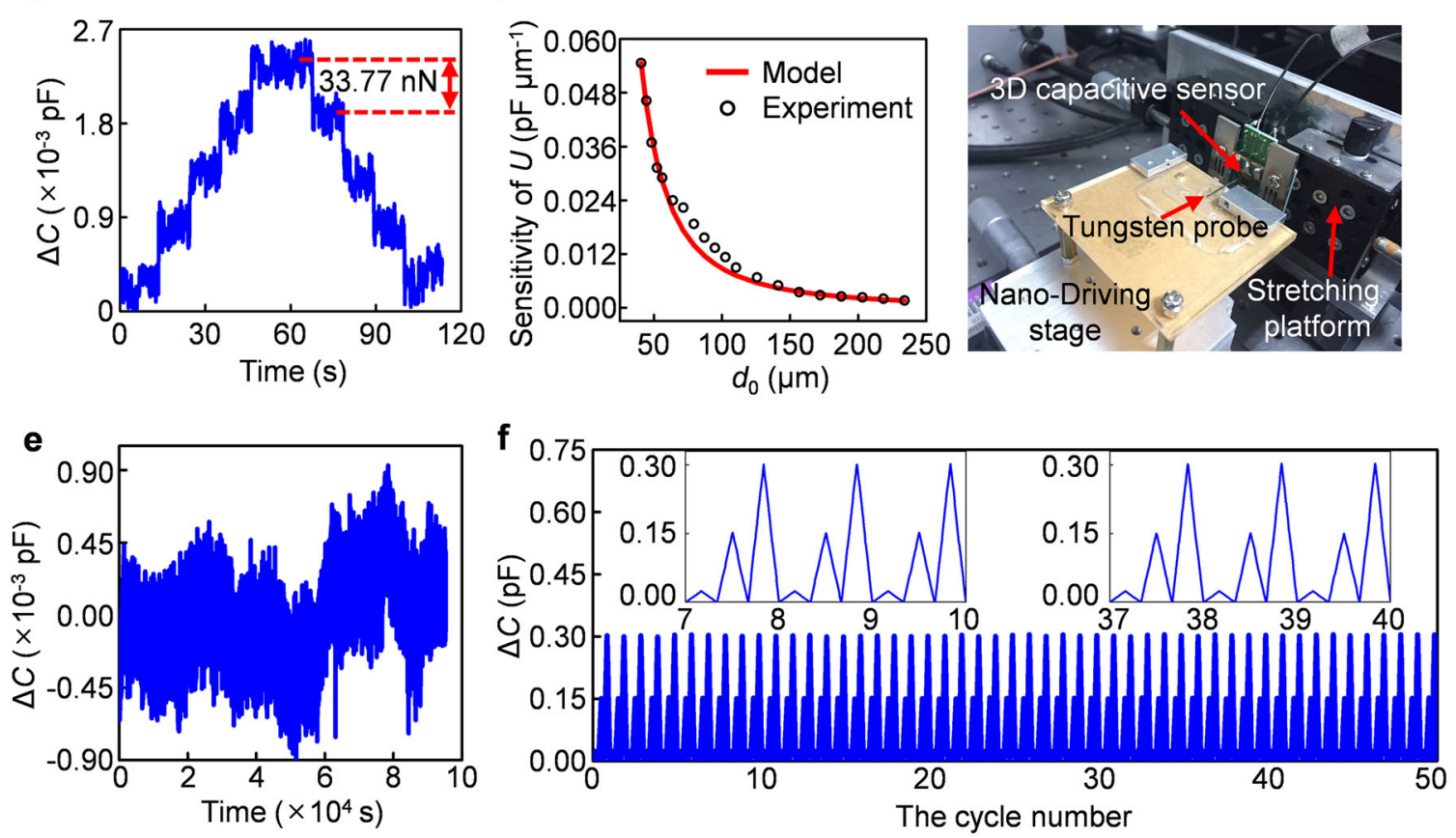

Fig. 3 Theoretical modeling and experimental measurements of the 3D capacitive sensor. a Schematic illustration of the deformation configuration for the 3D capacitive sensor under the out-of-plane compressive force loading. $\mathbf{b}$ Electrical response of a 3D capacitive sensor at different levels of compressive displacement $(U)$. The right panel shows the approximate linear correlation for relatively small compressive displacements $(<99 \mu \mathrm{m})$. c Response of a 3D capacitive sensor under the compressive loading applied by AFM (0.05 V for each increment voltage). The incremental force of adjacent steps is $33.77 \mathrm{nN}$. $\mathbf{d}$ Sensitivity of $Z$-direction displacement as a function of the initial gap $\left(d_{0}\right)$. The right panel shows the measurement system. e Variation of the output signal of a sample without external loads during 100,000 s. $\mathbf{f}$ Cyclic testing of the 3D capacitive sensor for 50 cycles. The initial gap between the upper and lower electrodes is tuned in sequence (from 2200 to $\sim 85$ to $\sim 54 \mu \mathrm{m}$ ) in an adjustment cycle. 
Table 2. Performance of three differential capacitance sensors by different fabrication methods.

\begin{tabular}{lllll}
\hline Name & Max. force resolution & Min. gap $(\mu \mathrm{m})$ & Force range $(\mu \mathrm{N})$ & Range of tunable sensitivity $(\%)$ \\
\hline MEMS differential capacitance sensor & $0.11 \mathrm{nN} @ 19 \mathrm{~Hz}$ & 6 & 24 & - \\
FT-S100 & $5 \mathrm{nN} @ 10 \mathrm{~Hz}$ & - & 100 & - \\
3D capacitive sensor & $5.22 \mathrm{nN} @ 19 \mathrm{~Hz}$ & 40.6 & 192.85 & 3300 \\
\hline
\end{tabular}

where the slope $a$ depends on the applied strain and geometric parameters of the cross-section of supporting ribbons (width $W_{s}$ and thickness $t$ ), which can be fitted by FEA results. For the basic parameters listed in Table 1, the coefficient $a=1085.4+$ $32753.1 \varepsilon_{\text {appl }}$ (see Supplementary Fig. 5 for details). According to the above analyses, a scaling law of the equivalent stiffness $(F / U)$ can be derived as $F / U=4 G W_{\mathrm{s}} t^{3} /\left[\left(1085.4+32753.1 \varepsilon_{\text {appl }}\right) W_{\mathrm{m}}^{2} L_{\mathrm{s}}\right]$. Figure $2 \mathrm{~d}$ presents the scaling laws, FEA results, and measurements of equivalent stiffness versus applied strain based on the geometric parameters listed in Table 1, where good agreements can be observed. The equivalent stiffness decreases with the increase of applied strain, namely greater variation of displacement under the action of unit force. The mechanical responses of the 3D seesaw-like mesostructure under compression are shown in Fig. 2e. The results shown in Fig. 2e (left) present a linear relationship between the compressive loads $(F)$ and compressive displacement $(U)$ of the indenter at five levels of applied strain. The right panel shows the $Z$ coordinates at unloading state and loading state when $\varepsilon_{\text {appl }}=0.8 \%$, where the mover is compressed until contacting the substrate, illustrating that the range of compressible displacement (or force) is determined by the initial gap $\left(d_{0}\right)$. At a relatively wide range (e.g., $\sim 20 \mathrm{mN}$ ) of the compressive force, the force still increases linearly with the displacement. The 3D seesaw-like mesostructure shows different force responses at the same compressive displacement due to the equivalent stiffness $(F / U)$ achieved by tuning the initial gap. Furthermore, the influence of supporting ribbon on equivalent stiffness $(F / U)$ is illustrated in Fig. $2 \mathrm{f}-\mathrm{h}$ and Supplementary Fig. 6 . The equivalent stiffness of $3 \mathrm{D}$ seesaw-like mesostructures increases with the reduction of length $\left(L_{s}\right)$, or the increases of width $\left(W_{s}\right)$ and thickness $(t)$. These results can serve as the design guidelines of the mechanically guided 3D assembly to achieve desired mechanical responses.

\section{Theoretical modeling and measurement for the tunable force sensing}

From the above mechanical analyses, the sensitivity and working range of the $3 \mathrm{D}$ capacitive sensor can be tuned by changing the initial gap between the mover and stators. Figure 3a illustrates the deformation configuration of the 3D capacitive sensor under a compressive force from the front view. The capacitance of 3D capacitive sensors can be expressed via the integration of Taylor series expansion as

$$
\begin{gathered}
\Delta C=C_{1}-C_{2} \\
=\varepsilon_{0} \varepsilon_{r} L_{m}\left[\frac{W_{m}^{2}}{4 d_{0}^{2}} \theta-\left(\frac{W_{m}^{2}}{6 d_{0}^{2}}-\frac{W_{m}^{4}}{32 d_{0}^{4}}\right) \theta^{3}+\left(\frac{W_{m}^{2}}{30 d_{0}^{2}}-\frac{W_{m}^{4}}{32 d_{0}^{4}}+\frac{W_{m}^{6}}{192 d_{0}^{6}}\right) \theta^{5}\right],
\end{gathered}
$$

where the permittivity of vacuum $\varepsilon_{0}=8.854187817 \times 10^{-12} \mathrm{~F} \mathrm{~m}^{-1}$, $C_{1}=\int_{0}^{\frac{W_{m}}{2}} \frac{\varepsilon_{0} \varepsilon_{\mathrm{r}} L_{\mathrm{m}} \cos \theta}{d_{0}-x \sin \theta} \mathrm{d} x$, and $C_{2}=\int_{-\frac{w_{m}}{2}}^{0} \frac{\varepsilon_{0} \varepsilon_{\mathrm{r}} L_{m} \cos \theta}{d_{0}-x \sin \theta} \mathrm{d} x$.

The measurements and analytical results for a $3 D$ capacitive sensor are shown in Fig. 3b, where the basic parameters are listed in Table 1 and the corresponding initial gap of the 3D capacitive sensor is $d_{0}=226 \mu \mathrm{m}$. It shows that the output signal $\left(S_{\mathrm{val}}\right)$ of the $3 \mathrm{D}$ capacitive sensor varies nonlinearly with the displacement during the process of out-of-plane compression (corresponding to a relatively large torsion angle). When the compressive displacement is below $\sim 99 \mu \mathrm{m}$ (around $d_{0} / 2$ ), the output signal depends linearly with the compressive displacement shown in the right panel of Fig. 3b, which can be predicted using the first term of Eq. (4) (light blue line). This linear relationship is beneficial to the use of developed sensors. On average, the modification of the output signal of the 3D capacitive sensor is around 333,910 with every picofarad (pF) change. Furthermore, the compressive forces are implemented on three typical cylindrical rods with diverse diameters $(D=145,120$, and $55 \mu \mathrm{m})$ through the 3D capacitive sensor, as shown in Supplementary Fig. 7. During the compression, the slope of output signal decreases with the increase of the initial gap (either from 80 to $200 \mu \mathrm{m}$ or from 80 to $320 \mu \mathrm{m}$ ), which indicates the tunability of 3D capacitive sensor.

Equations (3) and (4) indicate that the capacitance change of the $3 D$ capacitive sensor with a lower equivalent stiffness is greater than that with a higher equivalent stiffness for the same external force. In other words, the 3D capacitive sensor with a lower equivalent stiffness is more sensitive. Therefore, we adopt an optimized set of design parameters $\left(t=50 \mu \mathrm{m}, W_{\mathrm{s}}=400 \mu \mathrm{m}, L_{\mathrm{m}}=4 \mathrm{~mm}\right.$, and $W_{\mathrm{m}}=$ $5 \mathrm{~mm}$ ) for the 3D capacitive sensor, yielding a relatively low equivalent stiffness $\left(\sim 4.75 \mathrm{~N} \mathrm{~m}^{-1}\right)$. Firstly, the $3 \mathrm{D}$ capacitive sensor is measured by the atomic force microscope (AFM, MFP-3D Infinity; Oxford Instruments). By changing the applied voltage of AFM, different levels of compressive force were applied on the 3D capacitive sensor, as shown in Fig. 3c. The differential force of adjacent steps is about $33.77 \mathrm{nN}$, corresponding to $0.05 \mathrm{~V}$ for each increment of the applied voltage. Other differential forces (337.7, 67.54, and $50.65 \mathrm{nN}$ ), as shown in Supplementary Fig. 8a-c, correspond to the difference of the applied voltages of $0.5,0.1$, and $0.075 \mathrm{~V}$, respectively. The above calibration demonstrates that the developed sensor possesses a high force resolution, and ensures that the force can be distinguished at the level of nano-Newtons. To obtain the displacement sensitivity, the 3D capacitive sensor is loaded and unloaded under different levels of applied strains. Figure $3 \mathrm{~d}$ shows the measurements and theoretical results (Eq. (4)) of the displacement sensitivity versus the initial gap. The schematic illustration of the experimental system is shown in Fig. 3d (right), in which a tungsten probe is used to apply a compressive force. The variation of the output signal for two states $\left(d_{0} \approx 234 \mu \mathrm{m}\right.$ and $d_{0} \approx$ $40.6 \mu \mathrm{m})$ with a compressive displacement $(U=10 \mu \mathrm{m})$ is demonstrated in Supplementary Fig. 9. When the initial gap between mover and stators is reduced from $\sim 234$ to $\sim 40.6 \mu \mathrm{m}$, the corresponding displacement sensitivity is changed from $1.64 \times$

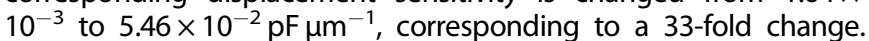
The experimental results are in good agreement with theoretical predictions. Theoretically, the displacement sensitivity can be improved further by reducing the gap according to Eq. (4). As shown in Supplementary Fig. 10, the force sensitivity and resolution of the developed sensor were calibrated via the commercial force sensor (FT-S100; FemtoTools). Supplementary Fig. 10c presents the calibrated results of the output signal versus compressive force, corresponding to the initial gap $d_{0}=40.6 \mu \mathrm{m}$. By applying the compressive force $F=8.82 \mu \mathrm{N}$, the output signal changes to $0.101 \mathrm{pF}$. Consequently, the force sensitivity of the 3D capacitive sensor can be determined as $1.15 \times 10^{-2} \mathrm{pF} \mu \mathrm{N}^{-1}$, and the ratio of displacement sensitivity-to-force sensitivity is $4.75 \mathrm{~N} \mathrm{~m}^{-1}$. The output signal noise of the sensor at a sampling frequency of $19 \mathrm{~Hz}$ is 20 . At this working state $\left(d_{0}=40.6 \mu \mathrm{m}\right)$, the available force range of the sensor is from 0 to $74.1 \mu \mathrm{N}$, and the force resolution can be calculated as $\sim 5.22 \mathrm{nN}^{69}$. Table 2 shows the performance 

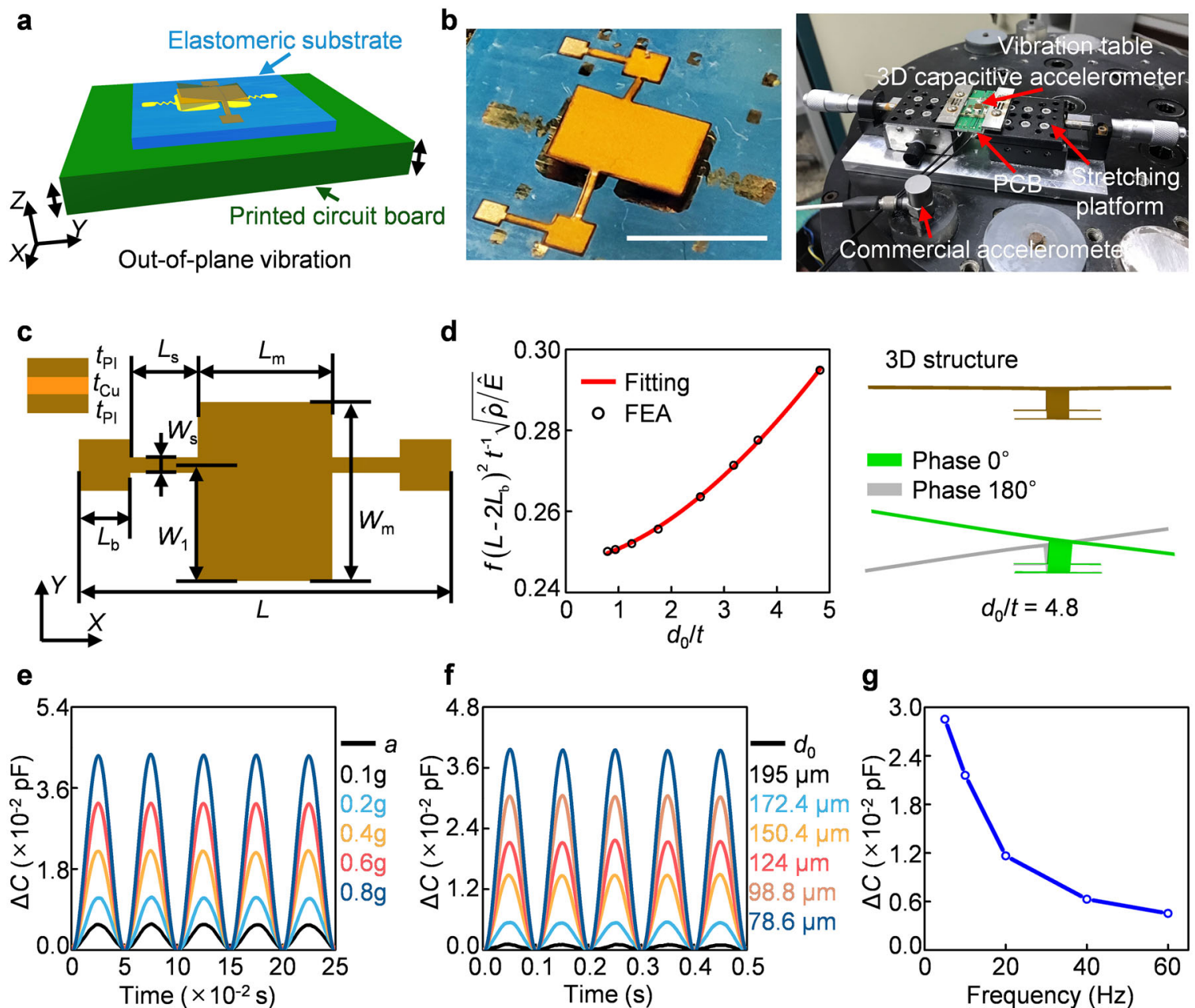

f

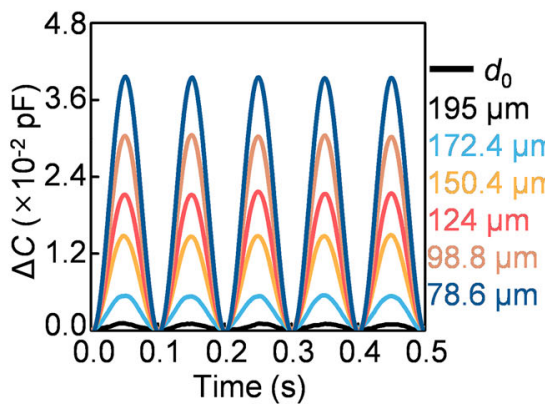

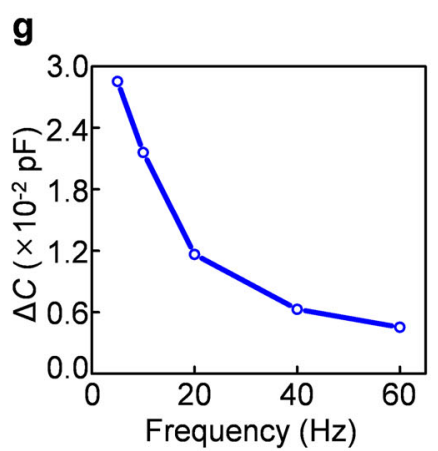

Fig. 4 Acceleration sensing with a tunable sensitivity. a Measurement schematic of 3D capacitive accelerometer. b Optical image of 3D capacitive accelerometer with the applied strain $\left(\varepsilon_{\mathrm{appl}}=0 \%\right)$ as shown in the left panel. The right panel shows the vibration test system. c Schematic illustration of geometric parameters of the 3D capacitive accelerometer with an asymmetric design. d The dimensionless resonant frequency $\left(f\left(L-2 L_{b}\right)^{2} t^{-1} \sqrt{\hat{\rho} / \hat{E}}\right)$ as a function of dimensionless gap $\left(d_{0} / t\right)$. The left panel shows the 3D mesostructure and out-ofplane vibration modes, corresponding to the largest amplitudes during vibration, i.e., green: phase $0^{\circ}$, gray: phase $180^{\circ}$. e Dynamic measurements of the 3D capacitive accelerometer with different levels of acceleration amplitude under a vibration frequency $(20 \mathrm{~Hz})$ and an initial gap $(124 \mu \mathrm{m})$. $\mathbf{f}$ Dynamic measurements of the 3D capacitive accelerometer with different initial gaps under a vibration signal $(0.2 \mathrm{~g}$, $10 \mathrm{~Hz})$. g Dynamic measurements of the 3D capacitive accelerometer with different excitation frequencies under an acceleration amplitude $(0.2 \mathrm{~g})$ and an initial gap $(124 \mu \mathrm{m})$. Scale bar, $4 \mathrm{~mm}$.

comparison among the developed 3D capacitive sensor, the MEMS differential capacitance force sensor ${ }^{69}$, and the commercial force sensor (FT-S100) ${ }^{69}$. The commercial force sensor adopts a combtooth capacitive structure, which has a maximum force resolution of $\sim 5 \mathrm{nN}$, a working range of $100 \mu \mathrm{N}$, and a sampling frequency of $10 \mathrm{~Hz}$ with a fixed force sensitivity. Here, the force resolution of the developed sensor is comparable to that of the commercial force sensor (FT-S100). Owing to the tunability, the working range of the developed sensor is much larger than that of FT-S100. Although the developed 3D capacitive sensor has a lower force resolution than that of MEMS differential capacitance force sensor, it achieves a tunability of force sensitivity and working range, thereby expanding the application range of a single capacitive sensor. It is noteworthy that a relatively higher level (e.g., $20 \%$ or $30 \%$ ) of prestretch can be also used in the $3 \mathrm{D}$ assembly to offer a higher stretchability of the 3D capacitive sensor, but the force sensitivity would be reduced with the use of higher prestretch (Fig. 3d). To meet the specific requirements of practical applications, the prestretch strain can be determined through a trade-off between the stretchability and sensitivity.
The long-term stability of the output signal is important for the practical application of force sensors. There are three samples of 3D capacitive sensors measured with a fixed applied strain, and the output signal of each sensor is recorded individually. As shown in Fig. 3e and Supplementary Fig. 11, the variation of the output signal of developed sensors without external loads is quite small during $100,000 \mathrm{~s}$, corresponding to a few changes of nano-Newton force, which indicates an excellent stability of 3D capacitive sensors. Figure $3 f$ presents the measurements of a 3D capacitive sensor under 50 loading-unloading cycles with three gaps ( 200, $\sim 85$, and $\sim 54 \mu \mathrm{m}$ in sequence) as one adjustment cycle corresponding to three different sensitivities. After switching between different working states for 50 cycles, the output signal remains unchanged, indicating that the $3 D$ capacitive sensors offer an excellent mechanical reliability. In order to verify the repeatability of the 3D capacitive sensor, the cyclic tests with three initial gaps $(\sim 200, \sim 85$, and $\sim 54 \mu \mathrm{m})$ were carried out, respectively, as shown in Supplementary Fig. 12. After 1000 cycles, the developed sensor shows good repeatability for different working states. 


\section{Acceleration sensing with a tunable sensitivity}

The design principle reported herein can be extended to the measurement of other physical quantities with a tunable sensitivity, such as acceleration. Here, a differential 3D capacitive accelerometer with tunable sensitivity fabricated by this strategy is demonstrated as an example. To enhance the sensitivity of the 3D capacitive accelerometer, an asymmetric design is adopted as shown in Fig. 4a. Figure 4b (left) and Supplementary Fig. 13 (SEM images) demonstrate the assembled configuration of 3D capacitive accelerometers. To avoid the vibration of the substrate, printed circuit boards (PCB) are placed under the substrate after adjusting the applied strains on the substrate, as shown in Fig. 4a and the right panel of $4 \mathrm{~b}$ (green part). The basic design parameters are shown in Fig. 4c and listed in Supplementary Table 3. The dynamic experimental system and the calibration results are shown in Fig. 4b (right) and Supplementary Fig. 14, respectively. In experiments, an out-of-plane vibration is produced by the vibration stage. The asymmetric design enables the excitation of the left-right vibration mode in the $Y-Z$ plane (Supplementary Fig. 15), and therefore, allows the acceleration sensing through measurement of the capacitation change. According to the FEA results shown in Fig. 4d, the resonant frequency $(f)$ of the sensor increases as the increment of the gap $\left(d_{0}\right)$, where $f=\hat{f} t\left(L-2 L_{\mathrm{b}}\right)^{-2} \sqrt{\hat{E} / \hat{\rho}}(\hat{E}$ is the effective modulus; $\hat{\rho}$ is the average density, see details in Supplementary note 1$). \hat{f}$ can be expressed by a function of the dimensionless gap $\left(d_{0} / t\right)$ as $\hat{f}\left(d_{0} / t\right)=\beta_{1}+\beta_{2}\left(d_{0} / t\right)^{\beta_{3}}$, where the $\beta_{i}$ can be determined by fitting the FEA results, as $\beta_{1}=0.247, \beta_{2}=0.00338$, and $\beta_{3}=1.68$. In Fig. $4 \mathrm{e}$, the $3 \mathrm{D}$ capacitive accelerometer can distinguish the different levels of acceleration amplitude (from 0.1 to $0.8 \mathrm{~g}$, where $\mathrm{g}$ is the gravitational acceleration) accurately with fixed vibration frequency $(20 \mathrm{~Hz})$ and initial gap $(124 \mu \mathrm{m})$. The output signal of the 3D capacitive accelerometer for different initial gaps is presented in Fig. 4f, with a vibration signal $(0.2 \mathrm{~g}, 10 \mathrm{~Hz})$ applied by the vibration table. As the initial gap decreases (from 195 to $78.6 \mu \mathrm{m})$, the corresponding sensitivity of the 3D capacitive accelerometer is improved from $5.59 \times 10^{-3}$ to $0.197 \mathrm{pF} \mathrm{g}^{-1}$ gradually. Compared with other accelerometers based on MEMS (Supplementary Table 4), the sensitivity of the 3D capacitive accelerometer shows a wider range of tunability. Figure $4 \mathrm{~g}$ shows the measurements under different excitation frequencies $(5,10$, $20,40$, and $60 \mathrm{~Hz})$ with the initial gap $(124 \mu \mathrm{m})$ and acceleration amplitude $(0.2 \mathrm{~g})$. As the excitation frequency of the vibration table increases, the amplitude of the output signal decreases gradually, due to the damping of the 3D capacitive accelerometer. Thereby, this developed accelerometer is applicable only to lowfrequency scenarios.

\section{DISCUSSION}

Guided by the rational designs based on the FEA and proposed theoretical model, this paper presents design and fabrication schemes of tunable 3D seesaw-like capacitive sensors. The 3D configurations of capacitive sensing systems can be switched rapidly and reversibly among various working states via the control of the applied strain on the elastomeric substrate, providing an effective route to adjust the device sensitivity. Combined computational and experimental studies showed the excellent performance of fabricated 3D capacitive sensor, as evidenced by the tunable range ( 33 times) of sensitivity unachievable previously. Moreover, an asymmetric capacitive sensor design enables the acceleration detection with a wider range of sensitivity than those reported in the literature. The demonstrated capabilities of tuning sensitivity and wide working range suggest promising applications in wearable electronics and biomedical devices.

\section{METHODS}

\section{Fabrication of developed sensors}

The fabrication of 3D capacitive sensors began with curing PDMS substrate $\left(\sim 1.6 \mathrm{~mm}\right.$; Sylgard 184 ; Dow) at $80^{\circ} \mathrm{C}$ for $3 \mathrm{~h}$. A commercial polyimide film $(\mathrm{PI}, \sim 100 \mu \mathrm{m})$ served as the shadow mask defined by laser cutting (VLS2.30; Universal Laser Systems). Then, thin layers of $\mathrm{Cr}(\sim 10 \mathrm{~nm})$ and $\mathrm{Au}(\sim 80 \mathrm{~nm})$ were deposited at certain locations on the PDMS substrate sequentially through the shadow mask that serves as lower electrodes (i.e., stators). Next, the preparation of 3D seesaw-like mesostructures in $\mathrm{PI} / \mathrm{Cu} / \mathrm{PI}$ began with laser cutting the $\mathrm{PI} / \mathrm{Cu} / \mathrm{PI}$ film $(\sim 75 \mu \mathrm{m})$ on a water-soluble tape (Water-Soluble Wave Solder $5414 ; 3 \mathrm{M}$ ) to define the 2D precursors, followed by depositing a thin layer of $\mathrm{Ti}(\sim 10 \mathrm{~nm})$ and $\mathrm{SiO}_{2}(\sim 50 \mathrm{~nm})$ at selective regions (i.e., bonding sites) by electron beam evaporation with a shadow mask. The prestretched PDMS substrates and the 2D structures were placed in a UV oven for 5 min to produce hydroxyl groups on both bonding sites and the top surface of the PDMS substrate. Then, the 2D structures were laminated onto the prestretched substrate by heating at $70^{\circ} \mathrm{C}$ for $10 \mathrm{~min}$, followed by the immersion in deionized water and washing the water-soluble tape. The release of prestrain in the substrate slowly completed the fabrication of 3D capacitive sensors.

\section{Measurements of developed sensors}

A custom-built uniaxial stretching machine was adopted as the assembly platform to apply the uniaxial strain to the PDMS substrate. To measure the differential capacitive signal of the 3D capacitive sensor, a customized electronic circuits was designed with all commercial components, including a universal capacitive readout chip (MS3110, Irvine Sensor) and a 24 bit ADC chip (AD7175-2, Analog Devices). The digital signal of the circuit is able to reach 24 bit resolution and $50 \mathrm{kHz}$ sampling rate. The digital signal of 3D capacitive sensor was recorded using the software LabVIEW. The difference between the digital signal of sensors with external loads and that without external loads was then converted into the capacitance change following the linear relationship in Fig. 3b. A mechanical test system (CB500; NANOVEA) was used to perform the compressive experiments. The experiments utilized a load-controlled

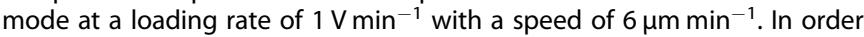
to calibrate the force responses of 3D capacitive sensors, the commercial force sensor (FT-S100; FemtoTools) and the AFM (MFP-3D; Oxford Instruments) were employed to implement different levels of forces. A nano-driving stage (S02893; Mad City Labs) was used to apply compressive displacement. A commercial accelerometer (352C33; PCB Piezotronics) was used to calibrate the performance of $3 \mathrm{D}$ capacitive accelerometer.

\section{Finite element analysis}

The commercial software ABAQUS was used to construct the FE models of 3D capacitive sensors and to analyze their mechanical performance under out-ofplane compressions. Four-node shell elements (S4R), eight-node solid elements (C3D8R), and analytic rigid were used to model the 3D seesawlike mesostructures, elastomer substrates, and indenter, respectively. The refined meshes were adopted to ensure the accuracy of numerical simulations. An ideally elastoplastic model was used for the copper (Cu) layer with Young's modulus $E_{\mathrm{Cu}}=119 \mathrm{GPa}$, Poisson's ratio $v_{\mathrm{Cu}}=0.34$, density $\rho_{\mathrm{Cu}}=8900 \mathrm{~kg} \mathrm{~m}^{-3}$, and yield stress $\sigma_{\text {yield_Cu}}=357 \mathrm{MPa}^{70}$. The material properties of polyimide (PI) layer are $E_{\mathrm{PI}}=2.5 \mathrm{GPa}, v_{\mathrm{PI}}=0.34$, and $\rho_{\mathrm{PI}}=$ $1420 \mathrm{~kg} \mathrm{~m}^{-3}$. The substrates exploited the Mooney-Rivlin constitutive model with $E_{\text {Substrate }}=1.8 \mathrm{MPa}, v_{\text {Substrate }}=0.48$, and $\rho_{\text {Substrate }}=1070 \mathrm{~kg} \mathrm{~m}^{-3}$.

\section{DATA AVAILABILITY}

The data that support the findings of this study are available from the corresponding authors upon reasonable request.

Received: 24 July 2021; Accepted: 16 September 2021; Published online: 08 October 2021

\section{REFERENCES}

1. Kim, K., Kim, B. \& Lee, C. H. Printing flexible and hybrid electronics for human skin and eye-interfaced health monitoring systems. Adv. Mater. 32, 1902051 (2020).

2. Lee, G. J., Choi, C., Kim, D.-H. \& Song, Y. M. Bioinspired artificial eyes: optic components, digital cameras, and visual prostheses. Adv. Funct. Mater. 28, 1705202 (2018). 
3. Yoo, Y. J. et al. Large-area virus coated ultrathin colorimetric sensors with a highly lossy resonant promoter for enhanced chromaticity. Adv. Sci. 7, 2000978 (2020).

4. Kim, B. et al. Rapid custom prototyping of soft poroelastic biosensor for simultaneous epicardial recording and imaging. Nat. Commun. 12, 3710-3710 (2021).

5. Ruth, S. R. A. et al. Rational design of capacitive pressure sensors based on pyramidal microstructures for specialized monitoring of biosignals. Adv. Funct. Mater. 30, 1903100 (2020).

6. Sheng, H. et al. A thin, deformable, high-performance supercapacitor implant that can be biodegraded and bioabsorbed within an animal body. Sci. Adv. 7, eabe3097 (2021).

7. Sim, K. et al. An epicardial bioelectronic patch made from soft rubbery materials and capable of spatiotemporal mapping of electrophysiological activity. Nat. Electron. 3, 775-784 (2020)

8. Jeong, J.-W. et al. Capacitive epidermal electronics for electrically safe, long-term electrophysiological measurements. Adv. Healthc. Mater. 3, 642-648 (2014).

9. Zhao, W. et al. Flexible transparent supercapacitors: materials and devices. Adv. Funct. Mater. 31, 2009136 (2021).

10. Liu, J., Yan, D. \& Zhang, Y. Mechanics of unusual soft network materials with rotatable structural nodes. J. Mech. Phys. Solids 146, 104210 (2021).

11. $\mathrm{Ma}, \mathrm{Q}$. et al. A nonlinear mechanics model of bio-inspired hierarchical lattice materials consisting of horseshoe microstructures. J. Mech. Phys. Solids 90, 179-202 (2016).

12. Ma, Q. \& Zhang, Y. Mechanics of fractal-inspired horseshoe microstructures for applications in stretchable electronics. J. Appl. Mech. 83, 111008 (2016).

13. Yan, D. et al. Soft three-dimensional network materials with rational bio-mimetic designs. Nat. Commun. 11, 1180 (2020).

14. Okamura, A. M. Haptic feedback in robot-assisted minimally invasive surgery. Curr. Opin. Urol. 19, 102-107 (2009).

15. Wan, C. et al. Artificial sensory memory. Adv. Mater. 32, 1902434 (2020).

16. $\mathrm{Yu}, \mathrm{Y}$. et al. Biofuel-powered soft electronic skin with multiplexed and wireless sensing for human-machine interfaces. Sci. Robot. 5, eaaz7946 (2020).

17. Gao, L. et al. Cellular carbon-film-based flexible sensor and waterproof supercapacitors. ACS Appl. Mater. Interfaces 11, 26288-26297 (2019).

18. Ershad, F. et al. Ultra-conformal drawn-on-skin electronics for multifunctional motion artifact-free sensing and point-of-care treatment. Nat. Commun. 11, 3823 (2020).

19. Byun, S.-H. et al. Design strategy for transformative electronic system toward rapid, bidirectional stiffness tuning using graphene and flexible thermoelectric device interfaces. Adv. Mater. 33, 2007239 (2021).

20. Kwak, J. W. et al. Wireless sensors for continuous, multimodal measurements at the skin interface with lower limb prostheses. Sci. Transl. Med. 12, eabc4327 (2020).

21. Song, E. et al. Miniaturized electromechanical devices for the characterization of the biomechanics of deep tissue. Nat. Biomed. Eng. 5, 759-771 (2021).

22. Han, M. et al. Catheter-integrated soft multilayer electronic arrays for multiplexed sensing and actuation during cardiac surgery. Nat. Biomed. Eng. 4, 997-1009 (2020).

23. Li, Y., Li, N., Oliveira, N. D. \& Wang, S. Implantable bioelectronics toward long-term stability and sustainability. Matter 4, 1125-1141 (2021).

24. Babaee, S. et al. Kirigami-inspired stents for sustained local delivery of therapeutics. Nat. Mater. 20, 1085-1092 (2021).

25. Pandit, M., Zhao, C., Sobreviela, G., Zou, X. \& Seshia, A. A high resolution differential mode-localized MEMS accelerometer. J. Microelectromech. Syst. 28, 782-789 (2019).

26. Jeong, $\mathrm{H}$. et al. Differential cardiopulmonary monitoring system for artifactcanceled physiological tracking of athletes, workers, and COVID-19 patients. Sci. Adv. 7, eabg3092 (2021).

27. Schwartz, G. et al. Flexible polymer transistors with high pressure sensitivity for application in electronic skin and health monitoring. Nat. Commun. 4, 1859 (2013).

28. Liu, Y. et al. Electronic skin from high-throughput fabrication of intrinsically stretchable lead zirconate titanate elastomer. Research 2020, 1085417 (2020)

29. Guan, Y.-S. et al. Air/water interfacial assembled rubbery semiconducting nanofilm for fully rubbery integrated electronics. Sci. Adv. 6, eabb3656 (2020).

30. Kim, M. K. et al. Soft-packaged sensory glove system for human-like natural interaction and control of prosthetic hands. NPG Asia Mater. 11, 43 (2019).

31. Lim, S. et al. Transparent and stretchable interactive human machine interface based on patterned graphene heterostructures. Adv. Funct. Mater. 25, 375-383 (2015).

32. Zhu, M. et al. Haptic-feedback smart glove as a creative human-machine interface (HMI) for virtual/augmented reality applications. Sci. Adv. 6, eaaz8693 (2020).

33. Song, J.-K. et al. Wearable force touch sensor array using a flexible and transparent electrode. Adv. Funct. Mater. 27, 1605286 (2017).

34. Luo, N. et al. Hollow-structured g raphene-silicone-com posite-based piezoresistive sensors: decoupled property tuning and bending reliability. Adv. Mater. 29, 1702675 (2017).
35. Han, M. et al. Three-dimensional piezoelectric polymer microsystems for vibrational energy harvesting, robotic interfaces and biomedical implants. Nat. Electron. 2, 26-35 (2019).

36. Askim, J. R., Mahmoudi, M. \& Suslick, K. S. Optical sensor arrays for chemical sensing: the optoelectronic nose. Chem. Soc. Rev. 42, 8649-8682 (2013).

37. $\mathrm{Wu}$, S. et al. Buckle-delamination-enabled stretchable silver nanowire conductors. ACS Appl. Mater. Interfaces 12, 41696-41703 (2020).

38. Cui, Z., Poblete, F. R. \& Zhu, Y. Tailoring the temperature coefficient of resistance of silver nanowire nanocomposites and their application as stretchable temperature sensors. ACS Appl. Mater. Interfaces 11, 17836-17842 (2019).

39. Niu, S. et al. A wireless body area sensor network based on stretchable passive tags. Nat. Electron. 2, 361-368 (2019).

40. Chang, Y. et al. First decade of interfacial iontronic sensing: from droplet sensors to artificial skins. Adv. Mater. 33, 2003464 (2021).

41. Won, S. M. et al. Multimodal sensing with a three-dimensional piezoresistive structure. ACS Nano 13, 10972-10979 (2019).

42. Zheng, Q., Lee, J.-h, Shen, X., Chen, X. \& Kim, J.-K. Graphene-based wearable piezoresistive physical sensors. Mater. Today 36, 158-179 (2020).

43. Wu, W., Wen, X. \& Wang, Z. Taxel-addressable matrix of vertical-nanowire piezotronic transistors for active and adaptive tactile imaging. Science 340, 952-957 (2013).

44. Shin, J. et al. Bioresorbable optical sensor systems for monitoring of intracranial pressure and temperature. Sci. Adv. 5, eaaw1899 (2019).

45. Ruth, S. R. A., Feig, V. R., Tran, H. \& Bao, Z. Microengineering pressure sensor active layers for improved performance. Adv. Funct. Mater. 30, 2003491 (2020).

46. Ruth, S. R. A. \& Bao, Z. Designing tunable capacitive pressure sensors based on material properties and microstructure geometry. ACS Appl. Mater. Interfaces 12, 58301-58316 (2020).

47. Li, C., Han, C., Zhao, Y., Anthony, C. \& Wei, X. Seesaw capacitive structure as an electrostatically actuated nonlinear impact resonator. Sens. Actuator A Phys. 315, 112279 (2020)

48. Fang, H. et al. Capacitively coupled arrays of multiplexed flexible silicon transistors for long-term cardiac electrophysiology. Nat. Biomed. Eng. 1, 0038 (2017).

49. Fu, M. et al. A highly sensitive, reliable, and high-temperature-resistant flexible pressure sensor based on ceramic nanofibers. Adv. Sci. 7, 2000258 (2020).

50. Liu, Q. et al. Highly transparent and flexible iontronic pressure sensors based on an opaque to transparent transition. Adv. Sci. 7, 2000348 (2020).

51. Puers, R. Capacitive sensors: when and how to use them. Sens. Actuator A Phys. 37-38, 93-105 (1993).

52. Yang, C. C. et al. Highly sensitive seesaw capacitive pressure sensor based on SOI wafer. Electron. Lett. 50, 376-377 (2014).

53. Sun, Y. \& Nelson, B. J. MEMS capacitive force sensors for cellular and flight biomechanics. Biomed. Mater. 2, S16 (2007).

54. Rotsch, C., Braet, F., Wisse, E. \& Radmacher, M. AFM imaging and elasticity measurements on living rat liver macrophages. Cell Biol. Int. 21, 685-696 (1997).

55. Cheng, J., Jia, Z. \& Li, T. Dielectric-elastomer-based capacitive force sensing with tunable and enhanced sensitivity. Extrem. Mech. Lett. 21, 49-56 (2018).

56. Je, C.H. et al. Sensitivity tunable capacitive type micro accelerometer. 2008 IEEE Sensors 2008, 1020-1023 (2008).

57. Peng, B. et al. A sensitivity tunable accelerometer based on series-parallel electromechanically coupled resonators using mode localization. J. Microelectromech. Syst. 29, 3-13 (2020).

58. Byun, S.-H. et al. Mechanically transformative electronics, sensors, and implantable devices. Sci. Adv. 5, eaay0418 (2019).

59. Zhang, F., Fan, Z. \& Zhang, Y. A theoretical model of postbuckling in straight ribbons with engineered thickness distributions for three-dimensional assembly. Int. J. Solids Struct. 147, 254-271 (2018).

60. Zhang, F. et al. Rapidly deployable and morphable 3D mesostructures with applications in multimodal biomedical devices. Proc. Natl Acad. Sci. USA 118, e2026414118 (2021)

61. Zhang, F., Liu, F. \& Zhang, Y. Analyses of mechanically-assembled 3D spiral mesostructures with applications as tunable inductors. Sci. China Technol. Sci. 62, 243-251 (2019).

62. Zhang, Y. et al. Printing, folding and assembly methods for forming 3D mesostructures in advanced materials. Nat. Rev. Mater. 2, 17019 (2017).

63. Bai, K. et al. Geometrically reconfigurable 3D mesostructures and electromagnetic devices through a rational bottom-up design strategy. Sci. Adv. 6, eabb7417 (2020).

64. Pang, W. et al. Electro-mechanically controlled assembly of reconfigurable 3D mesostructures and electronic devices based on dielectric elastomer platforms. Natl Sci. Rev. 7, 342-354 (2020).

65. $\mathrm{Xu}, \mathrm{S}$. et al. Assembly of micro/nanomaterials into complex, three-dimensional architectures by compressive buckling. Science 347, 154-159 (2015).

66. Zhang, $Y$. et al. A mechanically driven form of Kirigami as a route to $3 D$ mesostructures in micro/nanomembranes. Proc. Natl Acad. Sci. USA 112, 11757-11764 (2015) 
67. Fu, H. et al. Morphable 3D mesostructures and microelectronic devices by multistable buckling mechanics. Nat. Mater. 17, 268-276 (2018).

68. Ling, Y. et al. Laser-induced graphene for electrothermally controlled, mechanically guided, 3D assembly and human-soft actuators interaction. Adv. Mater. 32, 1908475 (2020)

69. Ye, J., Sun, T., Huang, D., Li, Z. \& Lin, L. Stand-alone differential capacitance force sensors with sub-nano-Newton sensitivity. J. Micromech. Microeng. 27, 095017 (2017).

70. Shi, Y. et al. Plasticity-induced origami for assembly of three dimensional metallic structures guided by compressive buckling. Extrem. Mech. Lett. 11, 105-110 (2017).

\section{ACKNOWLEDGEMENTS}

F.Z. acknowledges support from the National Natural Science Foundation of China (Grant 12002189) and the China Postdoctoral Science Foundation (Grant 2019M650649). Y.Z. acknowledges support from the National Natural Science Foundation of China (Grants 12050004 and 11921002), the Tsinghua National Laboratory for Information Science and Technology, the Henry Fok Education Foundation and the Institute for Guo Qiang, Tsinghua University (Grant 2019GQG1012). L.L. acknowledges support from National Natural Science Foundation of China (Grant 51675304). We thank Junshi Li, Kailun Xia, Zhanghui Wu, and Maosheng Chai for useful discussions.

\section{AUTHOR CONTRIBUTIONS}

Y.Z. and L.L. designed and supervised the research; Y.Z. and F.Z. led the structural designs, mechanics modeling and FEA predictions with assistance from Z.S., S.C., and T.J.; J.Y. led the fabrication and experimental characterization with assistance from F.Z., X.G., and Z.L.; Y.Z., L.L., F.Z., and J.Y wrote the text and designed the figures. All authors commented on the paper.

\section{COMPETING INTERESTS}

The authors declare no competing interests.

\section{ADDITIONAL INFORMATION}

Supplementary information The online version contains supplementary material available at https://doi.org/10.1038/s41528-021-00125-9.

Correspondence and requests for materials should be addressed to Fan Zhang, Li Lin or Yihui Zhang.

Reprints and permission information is available at http://www.nature.com/ reprints

Publisher's note Springer Nature remains neutral with regard to jurisdictional claims in published maps and institutional affiliations.

(C) Open Access This article is licensed under a Creative Commons Attribution 4.0 International License, which permits use, sharing, adaptation, distribution and reproduction in any medium or format, as long as you give appropriate credit to the original author(s) and the source, provide a link to the Creative Commons license, and indicate if changes were made. The images or other third party material in this article are included in the article's Creative Commons license, unless indicated otherwise in a credit line to the material. If material is not included in the article's Creative Commons license and your intended use is not permitted by statutory regulation or exceeds the permitted use, you will need to obtain permission directly from the copyright holder. To view a copy of this license, visit http://creativecommons. org/licenses/by/4.0/.

(c) The Author(s) 2021 